\title{
A nontraumatic myositis ossificans case of the forearm: Case report and literature review
}

\author{
SIMONA PĂTRU ${ }^{1 *}$, VLAD PĂDUREANU ${ }^{2 *}$, DUMITRU RĂDULESCU ${ }^{3 *}$, RADU RĂZVAN MITITELU $^{4}$, \\ RODICA PĂDUREANU ${ }^{4}$, MANUELA BĂCANOIU ${ }^{5}$ and DANIELA MATEI ${ }^{1}$ \\ Departments of ${ }^{1}$ Physical and Rehabilitation Medicine, ${ }^{2}$ Internal Medicine, ${ }^{3}$ Surgery, ${ }^{4}$ Biochemistry, \\ University of Medicine and Pharmacy of Craiova, Craiova 200349; \\ ${ }^{5}$ Department of Physical Therapy and Sports Medicine, University of Craiova, Craiova 200207, Romania
}

Received October 13, 2020; Accepted November 12, 2020

DOI: $10.3892 /$ etm.2021.9963

\begin{abstract}
Myositis ossificans (MO) is a rare, benign ossifying lesion characterized by focal formation of heterotopic bone and cartilage in extraskeletal soft-tissue that most commonly occurs in young adults. In most cases, no causative factor can be identified. The diagnosis of MO is usually based on the patient's history of trauma, clinical signs, on imaging appearance and histological examination. We present a non-traumatic MO case of the forearm in a 40-year-old man with weakness in left finger motion, a decrease in prehension for more than three weeks, without any weight loss, malaise, anorexia or fever. The clinical symptoms and radiological findings can be easily confused with malignant lesions. Treatment is usually conservative but, due to the limited strength and range of motion of the left hand, the tumor was extirpated and the diagnosis of MO was made by biopsy. The patient had no neurological deficits after surgical treatment and was discharged on the fifth day after the surgery in good condition with the recommendation to begin a rehabilitation program.
\end{abstract}

\section{Introduction}

Myositis ossificans (MO) is a rare, benign ossifying lesion characterized by focal formation of heterotopic bone and cartilage in extraskeletal soft-tissue, typically affected areas

Correspondence to: Dr Rodica Pădureanu, Department of Biochemistry, University of Medicine and Pharmacy of Craiova, 2-4 Petru Rares Street, Craiova 200349, Romania

E-mail: zegheanurodica@yahoo.com

Dr Manuela Băcănoiu, Department of Physical Therapy and Sports Medicine, University of Craiova, 146 Brestei Street, Craiova 200207, Romania

E-mail: manuelabacanoiu07@gmail.com

${ }^{*}$ Contributed equally

Key words: myositis ossificans, nontraumatic, histological diagnosis, forearm, clinical signs being the flexor muscles of the arm and the extensor muscles of the thigh (1).

MO most commonly occurs in young adults, with both males and females being affected equally (2). The etiology of $\mathrm{MO}$ is variable; in most cases, no causative factor can be identified. However, $\sim 60-70 \%$ of cases occur as a result of a repetitive minor mechanical trauma and other possible causative factors include ischemia, inflammation, infections, burns, neuromuscular disorders, hemophilia or drug abuse (1).

The MO localized form is usually a well-circumscribed lesion that frequently complicates hematoma formation of the muscles after sports trauma with contusions. The widespread form of MO occurs in progressive fibrodysplasia ossificans, a rare autosomal dominant mutation disease with ectopic calcifications in several muscles beginning in childhood (3).

The main differential diagnosis of MO is conducted with malignant tumors, such as osteosarcoma, soft-tissue sarcoma (4) or periarticular ossifications that usually occur in a context of central neurological pathologies (5).

The diagnosis of MO is usually based on the patient's history of trauma, clinical symptoms, on radiological findings and histological examination, while laboratory test results are usually normal (6).

Risk factors include male gender, past history of having formed heterotopic bone, hypertrophic osteoarthritis, ankylosing spondylitis, and diffuse idiopathic skeletal hyperostosis (7).

The typical clinical presentation of MO is as a significant inflammatory, rapidly growing, and painful muscular tumor that within a few weeks, becomes a firm and often painful mass which ossifies and becomes painless over 6-12 months.

When X-rays are performed two to three weeks after MO onset and sometimes even later, the ossifications are often missed because standard X-rays do not disclose any anomaly within the early stages of MO (8). A computerized tomography (CT) scan examination is more sensitive than X-ray for ossification diagnosis and may also show a central fatty metaplastic area (9). Magnetic resonance imaging (MRI) is the preferred diagnostic tool in the evaluation of a soft-tissue mass although the final diagnosis is always histological (10). Ultrasonography may be a sensitive imaging modality to early depict the acute phase in MO (10). 


\section{Case report}

A 40-year-old man was admitted in the Emergency County Hospital Craiova, Romania, Department of Physical and Rehabilitation Medicine, suffering from weakness in motion of the left fingers with a decrease in prehension for more than three weeks, with no history of a specific acute injury, or exercise-related trauma of the forearm. On physical examination, we palpated a single tumor, slightly tender, hard, not well-circumscribed, poorly mobile, painless lump on the volar side of the left distal forearm. The patient's significant medical history was negative and he reported no weight loss, malaise, anorexia or fever. The usual laboratory findings were normal. Written informed consent was obtained for patient participation and the publication of all associated data and images.

The T2-weighted MRI showed a hyperintense area with surrounding hypointense rim as a well-defined, inhomogeneous soft tissue mass, surrounded by a frame of lower signal intensity, signifying cortical calcification within the muscles of the flexor compartment in the distal and volar part of the left forearm.

The patient underwent a surgical procedure during which the tumor was extirpated completely and the specimen was sent to histopathological examination. The patient had no neurological deficits after surgical treatment and was discharged on the fifth day after the surgery in good condition with the recommendation to begin a rehabilitation program.

Tissue fragments were characterized by adipose tissue and striated muscle fibers dissociated by irregular, infiltrative area proliferating with a zonal pattern; the intermediate zone with osteoblasts including immature osteoid formations, surrounded by myxoid fibrous tissue; areas with fusiform cells with fasciculated growing pattern; richly vascularized areas; peripheral zone characterized of mature cartilage. No necrotic areas were noted.

The histological and pathological findings suggested the diagnosis of MO (Fig. 1A-D). Immunohistochemistry (IHC) tests were recommended. Upon IHC testing, cluster of differentiation (CD)34 was positive in vessels. CD68 was positive in intralesional rare macrophages. Anti- $\alpha$-smooth muscle actin ( $\alpha$-SMA) antibodies were positive in intralesional area and vessels. Anti-desmin antibodies were positive in striated muscle tissue. The activity of Ki67 proliferative index was positive in $1-3 \%$ of cells.

The patient started a program at the Physical Medicine and Rehabilitation Outpatient Clinic. He followed a 30-min exercise program performed five times/week, once daily, which involved active range-of-motion, hand and finger flexion and extension and ball resistance excercises. After 4 weeks of supervised program, he began an unsupervised home exercise program, $15 \mathrm{~min}$, twice a day, after he received detailed instructions concerning the type of exercises, repetitions, intensity, training, rest phases and demonstrations. After 12 weeks of rehabilitative treatment, we assessed the wrist flexion and extension with a goniometer and the Patient-Rated Wrist Evaluation (PRWE) a 15-item questionnaire designed to measure wrist pain and disability in activities of daily living (11). Extension increased to $62.7 \%$ and flexion achieved $63.2 \%$. Physical

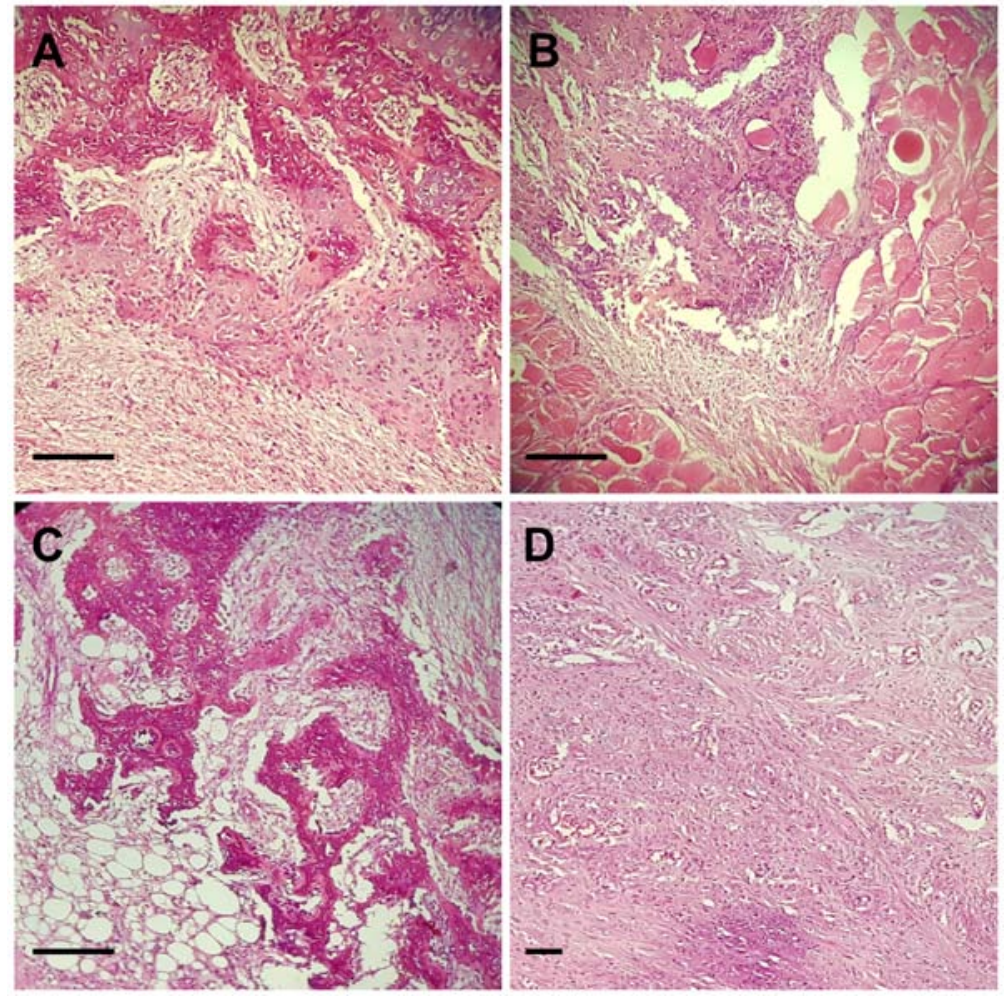

Figure 1. (A-D) Hematoxylin and eosin sections obtain from the tissue show (A) irregular bone and cartilage formation in a background of fasciitis-like, (B) osteoid trabecular entrapped by fragments of skeletal muscle, (C) a central zone of myositis ossificans showing fibroblastic/myofibroblastic proliferation in a (D) zonation pattern across the analyzed sections. Scale bar, $100 \mu \mathrm{m}$. 
Table I. Literature review of myositis ossificans: localization and etiology.

\begin{tabular}{|c|c|c|c|c|}
\hline Author, year (ref.) & Case no. & Age (years)/sex & Etiology & Localization \\
\hline Goto et al, 1998 (12) & 1 & $18 / \mathrm{F}$ & Repetitive minor trauma & Tip of the thumb \\
\hline Onen et al, 2019 (13) & 1 & $5 / \mathrm{M}$ & Nontrauma & Lumbar region \\
\hline Jayade et al, 2013 (14) & 1 & $25 / \mathrm{F}$ & Nontrauma & $\begin{array}{l}\text { Medial, lateral pterygoid, and } \\
\text { contralateral temporalis muscles }\end{array}$ \\
\hline Wei et al, 2015 (15) & 1 & $29 / \mathrm{F}$ & Long-term nape massage & Serratus anterior \\
\hline Akahane et al, 2015 (16) & 1 & $15 / \mathrm{F}$ & Nontrauma & Thenar region \\
\hline Simmonds et al, 2016 (17) & 1 & 5 months/F & $\begin{array}{l}\text { Nontrauma, nongenetic } \\
\text { mutation }\end{array}$ & Posterior triangle \\
\hline Lee et $a l, 2013$ (18) & 1 & $26 / F$ & Acupuncture & Paraspinal muscles of the neck \\
\hline Raudenbush et al, 2017 (19) & 1 & $30 / \mathrm{M}$ & Upper cervical spine fracture & Longus coli muscle \\
\hline Abdallah et al, 2014 (20) & 1 & 31/M & Nontrauma & Lumbar spine \\
\hline Bultheel et al, 2016 (21) & 1 & $21 / \mathrm{M}$ & Nontrauma & Superior anterolateral thigh \\
\hline Yunus et al, 2016 (22) & 1 & $36 / \mathrm{F}$ & Nontrauma & Hip \\
\hline Dubuisson et al, 2019 (23) & 1 & 5 years, 6 months/M & Nontrauma & Neck region \\
\hline
\end{tabular}

F, female; M, male.

therapy also increased hand function with better results to 12-week PRWE.

\section{Discussion}

In the event a patient has no history of traumatic injury, it is difficult to diagnose MO. In some cases, we must ask the patient concerning such minor injuries such as strenuous physical activity, heavy manual labor or weight lifting.

Most commonly MO affects the largest skeletal muscles of the body, typically after a trauma, but the exact pathophysiology is still poorly understand. We searched various MO cases of different localizations and different traumatic and nontraumatic etiology reported in the literature (Table I).

In 1998, Goto et al described a case of MO in a 18-year-old woman in the tip of the left thumb after repetitive minor trauma. The lesion arose in the subcutaneous fatty tissue in the distal portion of the thumb and had a typical zonal pattern (12). A 5-year-old pediatric patient who developed scoliosis associated with nontraumatic MO in the lumbar region was described by Onen et al (13). There has been no report of scoliosis associated with myositis ossificans. Jayade et al (14) described a rare case of MO in medial and lateral pterygoid and contralateral temporalis muscles in a 25-year-old woman without any obvious etiology, with no history of trauma, tooth extraction, or infection.

In 2015, Wei et al (15) presented the case of a 29-year-old woman with a rare form of MO of the serratus anterior that developed due to long-term aggressive nape massage. The symptoms disappeared after surgery. In addition, in 2015, the case of a 15-year-old Japanese girl with a 2-month history of a painful mass in the right thenar region without previous trauma was presented by Akahane et al. The diagnosis of MO was made on incisional biopsy (16).
In 2016, Simmonds et al presented a case of a 5-month-old infant with a posterior neck mass suspicious for neoplasia, which was treated with surgical resection and found to be a non-traumatic, non-genetic form of MO (17). In 2013, Lee et al (18) reported a case of a 26-year-old woman with MO in the paraspinal muscle of the neck after acupuncture. The patient was conservatively treated through rest and analgesics and the neck pain and swelling improved following several months.

Raudenbush et al (19) reported a case in 2017 of a 30-year-old male with upper cervical spine fracture occurring due to high-energy trauma that resulted in MO of the longus coli muscle. The patient was treated non-operatively for neck rotation and MO with gradual improvement of symptoms. Abdallah et al (20) presented the rare case of a 31-year-old Turkish man with MO not associated with trauma, with severe low back pain and restriction of low back motion. A biopsy was necessary to confirm diagnosis and the mass was surgically excised from the patient.

An atypical presentation of $\mathrm{MO}$ in the superior anterolateral thigh of a 21-year-old male is presented by Bultheel et al (21) in 2016. This case demonstrates that the diagnosis of MO can be more challenging in the absence of a history of trauma. In 2016, Yunus et al (22) presented a hip case of MO without any trauma occurring in a 36-year-old female. Nontraumatic MO is very rare in the literature. Dubuisson et al (23) in 2019 described a case of a 5 years and 6 months old boy with a cervical tumor causing torticollis and high suspicion of malignancy. The lesion was completely resected and the biopsy established the diagnosis of MO.

Involvement of the forearm is very rare and only a few cases have been reported to date. Say et al reported a rare case of MO on the forearm in a 10-year-old girl (24) and Grebić et al reported a case of MO in the forearm of a 48-year-old woman presenting clinically as a mesenchymal tumor (25). 
Early in the disease, the lesion is soft and painful, and within a few weeks, the lesion becomes firm; and over 12 months it ossifies and becomes painless. Significant functional deficits result in only $10-20 \%$ of patients (26).

MO passes through three characteristic phases. The acute phase (first week) is when the proliferation is composed of mesenchymal cells secreting a myxoid matrix, as well as fibroblasts exhibiting numerous mitoses, which gives the mass a pseudo-fibrosarcomatous appearance. The subacute phase (next two weeks) is when histologically fibroblasts differentiate into osteoblasts and secrete an osteoid matrix at the periphery of the initial myxoid zone, giving it a pseudo-osteosarcomatous appearance. Finally, the maturation phase (2-5 weeks) is when a histological diagnosis can accurately be carried out (27).

Due to the presence of bone formation as well as a similar epidemiology, osteosarcoma needs to be excluded. It is very important to identify the early stage of MO using imaging. However, early in the disease course, radiographs are often negative and a biopsy conducted at the early stage of MO may lead to a wrong diagnosis of sarcoma. On the other hand, when biopsy is delayed, a true sarcoma may be missed. Computerized axial tomography optimally identifies the typical patterns of this disease, including the separation of the mass from the adjacent cortex and the decreased attenuation of the center of the mass (9).

MRI is the elected investigation for evaluating soft-tissue lesions with the classic finding for $\mathrm{MO}$, a peripheral rim enhancement that correlates with calcification and ossification (28). MO can disappear spontaneously, the treatment is usually reserved for symptomatic lesions: Rest, ice, compression, nonsteroidal anti-inflammatory drug (NSAID) therapy could be initiated leading to clinical improvement and concomitant decrease of the soft tissue swelling. The specialty literature recognized that NSAIDs may stop the evolutionary process of MO (29).

Surgical excision is generally reserved for symptomatic MO lesions. However, since recurrence has been reported, excision with clear resection margins is recommended (30). When symptoms are not associated with trauma, the diagnosis of MO is challenging. The MRI findings may suggest the mesenchymal tumor like malignant fibrous histiocytoma.

Finally, diagnosis is always established by histopathological examination. It may be difficult with histological evidence alone to differentiate an MO from a sarcoma; therefore, correlation of the clinical and radiological findings is important in such cases. There is no need for further therapy once the diagnosis of MO has been established by excision. MO is a rare clinical entity and understanding its etiology and pathophysiology can save the patient from the anxiety of a suspected neoplasm.

In conclusion, particularities of our case report is the diagnosis of MO in middle aged patients, which is very rare, as MO commonly occurs in young males. Our patient had no history of acute injury or repetitive minor trauma of the forearm, thus the aetiology still remains unclear. In addition, involvement of the forearm muscles is rare. We found in our literature search only a few cases reported. Treatment is usually conservative but, due to the limited strength and range of motion of the left hand, the tumor was extirpated and the diagnosis of MO was made by biopsy. In future research, the molecular mechanisms of this rare disease must be discovered and gene therapy may be used in the early stages as a treatment strategy.

\section{Acknowledgements}

Not applicable.

\section{Funding}

No funding was received.

\section{Availability of data and materials}

Further information regarding the case and the review may be requested from the corresponding author upon reasonable request.

\section{Authors' contributions}

DR performed the surgical procedure. SP, VP, RRM, RP, and DM carried out the patient investigation and SP, VP, RP, and MB data curation. SP, VP, DR, and DM carried out the writing and original draft preparation. VP, RRM, RP, DM and SP performed the literature data review and SP and VP finally reviewed the manuscript. All authors have read and agreed to the published version of the manuscript.

\section{Ethics approval and consent to participate}

The case report was approved by the local institutional Ethics Committee of the University of Medicine and Pharmacy of Craiova.

\section{Patient consent for publication}

Written informed consent was obtained for patient participation and the publication of all associated data and images.

\section{Competing interests}

The authors declare that they have no competing interests.

\section{References}

1. Walczak BE, Johnson CN and Howe BM: Myositis ossificans. J Am Acad Orthop Surg 23: 612-622, 2015.

2. Li PF, Lin ZL and Pang ZH: Non-traumatic myositis ossificans circumscripta at elbow joint in a 9-year old child. Chin J Traumatol 19: 122-124, 2016.

3. Bridges AJ, Hsu KC, Singh A, Churchill R and Miles J: Fibrodysplasia (myositis) ossificans progressiva. Semin Arthritis Rheum 24: 155-164, 1994.

4. Mahale YJ, Vyawahare CS, Dravid NV, Upase A and Rathi R: A rare case of non traumatic myositis ossificans circumscripta. J Orthop Case Rep 5: 15-17, 2015.

5. Olsen KM and Chew FS: Tumoral calcinosis: Pearls, polemics, and alternative possibilities. Radiographics 26: 871-885, 2006.

6. Man SC, Schnell CN, Fufezan O and Mihut G: Myositis ossificans traumatica of the neck-a pediatric case. Maedica (Buchar) 6: 128-131, 2011.

7. Aneiros-Fernandez J, Caba-Molina M, Arias-Santiago S, Ovalle F, Hernandez-Cortes P and Aneiros-Cachaza J: Myositis ossificans circumscripta without history of trauma. J Clin Med Res 2: 142-144, 2010. 
8. Goldman AB: Myositis ossificans circumscripta: A benign lesion with a malignant differential diagnosis. AJR Am J Roentgenol 126: 32-40, 1976.

9. Amendola MA, Glazer GM, Agha FP, Francis IR, Weatherbee L and Martel W: Myositis ossificans circumscripta: Computed tomographic diagnosis. Radiology 149: 775-779, 1983.

10. Wang H, Nie P, Li Y, Hou F, Dong C, Huang Y and Hao D: MRI findings of early myositis ossificans without calcification or ossification. Biomed Res Int 2018: 4186324, 2018.

11. Kleinlugtenbelt YV, Krol RG, Bhandari M, Goslings JC, Poolman RW and Scholtes VA: Are the patient-rated wrist evaluation (PRWE) and the disabilities of the arm, shoulder and hand (DASH) questionnaire used in distal radial fractures truly valid and reliable? Bone Joint Res 7: 36-45, 2018.

12. Goto H, Hatori M, Kokubun S and Makino M: Myositis Ossificans in the tip of the thumb: A case report. Tohoku J Exp Med 184: 67-72, 1998.

13. Onen MR, Varol E, Tosun MI and Naderi S: Nontraumatic myositis ossificans as an uncommon cause of scoliosis: Case report and review of the literature. World Neurosurg 123: 208-211, 2019.

14. Jayade B, Adirajaiah S, Vadera H, Kundalaswamy G, Sattur AP and Kalkur C: Myositis ossificans in medial, lateral pterygoid, and contralateral temporalis muscles: A rare case report. Ora Surg Oral Med Oral Pathol Oral Radiol 116: E261-E266, 2013.

15. Wei J, Jia Y and Liang B: Myositis ossificans of the serratus anterior as a rare complication of massage: A case report. J Med Case Rep 9: 143, 2015.

16. Akahane T, Mori N and Nakatsuchi Y: Myositis ossificans occupying the thenar region: A case report. J Med Case Rep 9: 105, 2015 .

17. Simmonds J, Taki N, Chilton I and Vecchiotti M: A rare case of pediatric nontraumatic myositis ossificans in the posterior triangle. Int J Pediatr Otorhinolaryngol 84: 116-118, 2016.

18. Lee DG, Lee SH, Hwanga SW, Kim ES and Eoh W: Myositis ossificans in the paraspinal muscles of the neck after acupuncture: A case report. Spine J 13: e9-e12, 2013.

19. Raudenbush BL, McCalla D, Mesfin A and Rubery PT: Myositis ossificans of the longus coli muscle following cervical spine fracture-dislocation. J Spinal Cord Med 40: 372-376, 2017.
20. Abdallah A, Gokcedag A, Ofluoglu AE and Emel E: Non-traumatic myositis ossificans in the lumbar Spine. Am J Case Rep 15: 421-425, 2014

21. Bultheel M, Kirby JH, Viljoen JT and Viviers PL: An atypical presentation of myositis ossificans. SA J Sports Med 28: 33-34, 2016.

22. Yunus O, Ozcan MS, Sezer HB, Kilinc BE and Eren OT: Nontraumatic myositis ossificans of hip: A case presentation. Case Rep Orthop 2016: 1982656, 2016.

23. Dubuisson A, Lombard A and Otto B: Pseudomalignant myositis ossificans of the neck in a child: Case report and review of the literature. World Neurosurg 130: 95-97, 2019.

24. Say F, Coskun S, Buelbuel M and Alici O: Myositis ossificans on the forearm in a 10-year-old girl. J Pediatr Orthop B 24: 223-225, 2015.

25. Grebić D, Pozderac I, Milas I and Eljuga D: A sporadic case of myositis ossificans of the forearm presenting clinically as mesenchimal tumor. Medicina fluminensis 53: 225-230, 2017.

26. Rothwell AG: Quadriceps hematoma: A prospective clinical study. Clin Orthop Relat Res: 97-103, 1982.

27. McCarthy EF and Sundaram M: Heterotopic ossification: A review. Skeletal Radiol 34: 609-619, 2005.

28. De Smet AA, Norris MA and Fisher DR: Magnetic resonance imaging of myositis ossificans: Analysis of seven cases. Skeletal Radiol 21: 503-507, 1992.

29. Mann D and McCormack B: Commentary: Myositis ossificans. CJEM 1: 199, 1999.

30. Mavrogenis AF, Soucacos PN and Papagelopoulos PJ: Heterotopic ossification revisited. Orthopedics 34: 177, 2011. International (CC BY-NC-ND 4.0) License. 